\title{
All Polytopes Are Quotients, and Isomorphic Polytopes Are Quotients by Conjugate Subgroups
}

\author{
M. I. Hartley* \\ University of Western Australia, \\ Nedlands, WA 6907, Australia \\ hartley@maths.uwa.edu.au
}

\begin{abstract}
In this paper it is shown that any (abstract) polytope $\mathcal{P}$ is a quotient of a regular polytope $\mathcal{M}$ by some subgroup $N$ of the automorphism group $W$ of $\mathcal{M}$, and also that isomorphic polytopes are quotients of $\mathcal{M}$ by conjugate subgroups of $W$. This extends work published in 1980 by Kato, who proved these results for a restricted class of polytopes which he called "regular". The methods used here are more elementary, and treat the problem in a purely nongeometric manner.
\end{abstract}

\section{Introduction}

The main results of this paper are stated (roughly) in its title. The proofs of these results require some basic knowledge of abstract polytopes and Coxeter groups, so these are defined and explored briefly later in this section. First, the remainder of this paper's contents is outlined. Section 2 shows a way to construct a partially ordered set from a string C-group and one of its subgroups, the plan being to show eventually that any polytope is isomorphic to such a poset. It is noted that these posets are quotients of regular polytopes. Following the plan, Section 3 introduces the flag action of a C-group on the set of flags of a polytope, and some properties required for the main theorems are derived. The flag action is critical to the results of this paper, and was studied in [4]. Section 4 studies isomorphisms between polytopes, and how they interact with the flag action. In Section 5 it is shown how, given a polytope and a C-group acting via the flag action, we may find a subgroup which can be used in the quotient construction to yield a poset isomorphic to the original polytope. It is also shown that if this is done on two

\footnotetext{
* Current Address: Sepang Institute of Technology, Level 5, Klang Parade, 2112 Jalan Meru, 41050 Klang, Selangor Darul Ehsan, Malaysia. hartleym@sit.edu.my.
} 
polytopes, the subgroups so obtained will be conjugate if and only if the polytopes are isomorphic.

The definition of an abstract polytope used here follows that of the incidence polytopes of [2], [7], [8], [10] and others-namely, that an $n$-incidence polytope $\mathcal{P}$ is a partially ordered set with a strictly monotone rank function $\operatorname{rank}_{\mathcal{P}}: \mathcal{P} \rightarrow\{-1,0, \ldots, n\}$, such that $\mathcal{P}$ satisfies certain other properties, listed below. Let $\mathcal{P}_{i}=\{F \in \mathcal{P}: \operatorname{rank} F=i\}$. We require firstly that $\left|\mathcal{P}_{-1}\right|=\left|\mathcal{P}_{n}\right|=1$ (and we use the notation $\mathcal{P}_{-1}=\left\{P_{-1}\right\}$ and $\mathcal{P}_{n}=\left\{P_{n}\right\}$ ). The totally ordered subsets of $\mathcal{P}$ are called chains, and the maximal chains, flags. Secondly, we require that every flag has exactly $n+2$ elements, that is, one from each $\mathcal{P}_{i}$. Thirdly, we require $\mathcal{P}$ to be strongly flag connected, that is, for any two flags $\Phi$ and $\Phi^{\prime}$ of $\mathcal{P}$, there exists a sequence $\Phi=\Phi_{0}, \Phi_{1}, \ldots, \Phi_{m}=\Phi^{\prime}$ of flags of $\mathcal{P}$, such that, for $1 \leq i \leq m$, the flags $\Phi_{i-1}$ and $\Phi_{i}$ differ by exactly one element of $\mathcal{P}$, and $\Phi \cap \Phi^{\prime} \subseteq \Phi_{i}$. Fourthly and lastly, we require that, for $F, G \in \mathcal{P}$ with $F \leq G$ and $\operatorname{rank}_{\mathcal{P}} F+1=i=\operatorname{rank}_{\mathcal{P}} G-1$, there should exist exactly two elements $H$ of $\mathcal{P}_{i}$ with $F \leq H \leq G$. If there is no danger of ambiguity, the subscript $\mathcal{P}$ of $\operatorname{rank}_{\mathcal{P}}$ may be dropped.

Denote the set of flags of $\mathcal{P}$ by $\mathcal{F}(\mathcal{P})$, and, for any $\Phi \in \mathcal{F}(\mathcal{P})$, let $(\Phi)_{i}$ denote its element of rank $i$. A corollary to our last condition on $\mathcal{P}$ is that, for any $\Phi \in \mathcal{F}(\mathcal{P})$ and for $0 \leq i \leq n-1$, the set $\left\{\Phi^{\prime} \in \mathcal{F}(\mathcal{P}):\left(\Phi^{\prime}\right)_{j}=(\Phi)_{j}\right.$ for all $\left.j \neq i\right\}$ contains exactly two flags of $\mathcal{P}$, one of them $\Phi$ itself. It may be shown easily from this definition that a 1-polytope has exactly four elements, and that any 2 -polytope is an $l$-gon, for some $l \geq 2$, possibly infinite.

For $F, G \in \mathcal{P}$ with $F \leq G$, the set $\mathcal{S}=G / F=\{H \in \mathcal{P}: F \leq H \leq G\}$ is called a section of $\mathcal{P}$. If $\operatorname{rank} F=i$ and $\operatorname{rank} G=j$, then $\mathcal{S}$ will be a $(j-i-1)$-incidence polytope, and we call it a $(j-i-1)$-section of $\mathcal{P}$. The rank function $\operatorname{rank}_{\mathcal{S}}$ for $\mathcal{S}$ will be related to that for $\mathcal{P}$ via $\operatorname{rank}_{\mathcal{S}} H=\operatorname{rank}_{\mathcal{P}} H-\operatorname{rank}_{\mathcal{P}} F-1$, for all $H \in \mathcal{S}$.

A section of the form $F / F_{-1}$, where rank $F=i$, is called an $i$-face of $\mathcal{P}$, as is the element $F$ itself. The $(n-1)$-faces of $\mathcal{P}$ are called facets. Also, the sections of the form $P_{n} / F$ are called figures of $\mathcal{P}$, or vertex figures if $\operatorname{rank}_{\mathcal{P}} F=0$.

This is all the basic information about polytopes that we shall need. The main results of this paper are Theorem 5.2, that any polytope is isomorphic to a quotient, and Theorem 5.3, that if two such quotients are isomorphic, they are quotients by conjugate subgroups. Kato [6], and later Davis [3], obtained these results for those polytopes $\mathcal{P}$ such that, for any two flags $\Phi$ and $\Phi^{\prime}$ of $\mathcal{P}$ and for $0 \leq i \leq n-2$, the sections $(\Phi)_{i+2} /(\Phi)_{i-1}$ and $\left(\Phi^{\prime}\right)_{i+2} /\left(\Phi^{\prime}\right)_{i-1}$ are isomorphic. Davis's methods were very different from Kato's, being similar to those used by Tits [11] to obtain similar results in the theory of buildings. This paper shows that no restriction on a polytope's 2-sections is needed.

To understand the constructions and proofs that are to follow, a cursory understanding of Coxeter group theory is also necessary. A Coxeter group is a group of the form $W=\left\langle s_{0}, \ldots, s_{n-1}: s_{i}^{2}=\left(s_{i} s_{j}\right)^{p_{i, j}}=1\right\rangle$, where the $p_{i, j} \geq 2$ are elements of $\mathbf{Z}^{+} \cup\{\infty\}$. If for some $i$ and $j$ we have $p_{i, j}=\infty$, there is deemed to be no relation of the form $\left(s_{i} s_{j}\right)^{p}=1$ in the definition of $W$.

Let $S=\left\{s_{0}, \ldots, s_{n-1}\right\}$. For any Coxeter group $W$, with generating set $S$, we define the Coxeter graph $G(W, S)$ of $W$ to be the graph with vertex set $S$, and with an edge between $s_{i}$ and $s_{j}$ whenever $p_{i, j} \geq 3$. If also $p_{i, j} \geq 4$, we label this edge $p_{i, j}$.

The subgroups of $W$ generated by subsets of $S$ are called the (standard) parabolic 
subgroups, and have a number of nice properties. For $I \subseteq S$, denote $\langle I\rangle$ by $W_{I}$. Then $W_{I}$ is a Coxeter group with generating set $I$, and its Coxeter graph $G\left(W_{I}, I\right)$ is the induced subgraph of $G(W, S)$ having $I$ as its vertex set. Another important property is the so-called intersection property, that $W_{I} \cap W_{J}=W_{I \cap J}$. For these and other less basic results about Coxeter groups, see [1] or [5]. Other groups also have the intersection property. In fact, if a group $\left\langle s_{0}, \ldots, s_{n-1}\right\rangle$ has this property, it is called a $C$-group. The definition above of the Coxeter graph may also be applied to an arbitrary C-group.

\section{Quotients}

Let $W$ be a string C-group. By this, we mean a C-group whose graph is a path, so $p_{i, j}=2$ whenever $i \neq j, j \pm 1$. We set $q_{i}=p_{i-1, i}$. Such a C-group is said to be of type $\left[q_{1}, \ldots, q_{n-1}\right]$, and is a quotient of the Coxeter group of this type. The $q_{i}$ are (as usual) permitted to be infinite. If $S=\left\{s_{0}, \ldots, s_{n-1}\right\}$ is the generating set for $W$, then let $H_{i}$ be the parabolic subgroup $\left\langle s_{j}: j \neq i\right\rangle$. It will be useful also to define $W_{<i}=\left\langle s_{j}: j<i\right\rangle$ and $W_{>i}=\left\langle s_{j}: j>i\right\rangle$. Note that because of the form of the C-group $W$ under consideration, we have $H_{i}=W_{<i} W_{>i}=W_{>i} W_{<i}$.

Given a subgroup $N$ of $W$, we construct our partially ordered set $\mathcal{Q}$, from the double cosets $N w H_{i}, w \in W$. We therefore need to know some of the properties of these double cosets. Note first that, for $u, v \in W, N u H_{i}=N v H_{i}$ if and only if $u \in N v H_{i}$. We also have the following.

Lemma 2.1. If $N u H_{i}=N v H_{j}$ for any $j \neq i$, then $N u H_{i}=W$.

Proof. Now $u \in N v H_{j}$, so $N u H_{i}=N v H_{j}=N u H_{j}$. Multiplying on the right by $H_{j} H_{i}$ yields $N u H_{i} H_{j} H_{i}=N u H_{j} H_{i}=N u H_{i} H_{i}=N u H_{i}$. An inductive argument would then show that $N u H_{i}=N u\left(H_{i} H_{j}\right)^{k} H_{i}$ for all $k \geq 1$. Now, for any $w \in W$, we can write $u^{-1} w=v_{1} s_{i} v_{2} s_{i} \cdots v_{m} s_{i} v_{m+1}$ for some $m \in \mathbf{Z}^{+}$, where each $v_{k}$ is an element of $\left\langle s_{j}: j \neq i\right\rangle=H_{i}$. Thus $u^{-1} w \in\left(H_{i} H_{j}\right)^{m} H_{i}$ (since $s_{i} \in H_{j}$ ), and so $w \in u\left(H_{i} H_{j}\right)^{m} H_{i}$, which is a subset of $N u H_{i}$. It follows that $W \subseteq N u H_{i}$, yielding $W=N u H_{i}$ as required.

Let $\mathcal{Q}_{i}=\left\{N w H_{i}: w \in W\right\}$, for $0 \leq i \leq n-1$. Also, let $\mathcal{Q}_{-1}$ and $\mathcal{Q}_{n}$ be single element sets, say $\left\{Q_{-1}\right\}$ and $\left\{Q_{n}\right\}$, respectively. Let $\mathcal{Q}$ be the "disjoint union" of the $\mathcal{Q}_{i}$, and equip it with the relation " $\leq$ " defined by setting $Q_{-1} \leq X \leq Q_{n}$ for all $X \in \mathcal{Q}$, and $N u H_{i} \leq N v H_{j}$ if and only if $i \leq j$ and $N u H_{i} \cap N v H_{j}$ is nonempty.

By disjoint union in this context, it is intended that the sets $\mathcal{Q}_{i}$ be treated as disjointeven if, in a strict set-theoretic sense, they are not. We could accomplish this by labelling each element of $\mathcal{Q}_{i}$ with the number $i$ of the set it came from (so then $N u H_{i}$ would become the ordered pair $\left.\left(N u H_{i}, i\right)\right)$ but this is notationally very messy. In any event, we are not interested in the case where this need arises, for if $N u H_{i}=N v H_{j}$ for some $u, v \in W$ and $i \neq j$, then the set $\mathcal{Q}=\bigcup_{i=-1}^{d}\left(\mathcal{Q}_{i} \times\{i\}\right)$ cannot form a polytope, since the set $\{K: F<K<G\}$, where $\operatorname{rank} F+1=\operatorname{rank} G-1=i$ and $F<G$, would have only one element, $(W, i)$. 
We have not yet shown that " $\leq$ " is a partial order. We proceed to do so. The following lemma is useful.

Lemma 2.2. Let $0 \leq i_{1}<i_{2}<\cdots<i_{m} \leq n-1$, and let $u_{i_{1}}, \ldots, u_{i_{m}} \in W$ be such that, for any $j \neq m,\left(N u_{i_{j}} H_{i_{j}}\right) \cap\left(N u_{i_{j+1}} H_{i_{j+1}}\right)$ is nonempty. Then there exists some $u \in W$ such that $N u_{i_{j}} H_{i_{j}}=N u H_{i_{j}}$ for all $j$.

Proof. By induction on $m$. If $m=1$, there is nothing to prove, and if $m=2$, letting $u \in$ $N u_{i_{1}} H_{i_{1}} \cap N u_{i_{2}} H_{i_{2}}$ yields the result. Now, let $k \geq 3$ and assume that the lemma is true if $m<k$. Let $m=k$. Since the sequence $N u_{i_{2}} H_{i_{2}}, \ldots, N u_{i_{m}} H_{i_{m}}$ satisfies all the conditions of the lemma, yet has less than $k$ elements, we deduce that there exists $u^{\prime} \in W$ such that $N u_{i_{j}} H_{i_{j}}=N u^{\prime} H_{i_{j}}$ for $j \geq 2$. Likewise, there exists $v \in W$ such that $N u_{i_{j}} H_{i_{j}}=N v H_{i_{j}}$ for all $j \leq 2$. In particular, note that $N v H_{i_{2}}=N u^{\prime} H_{i_{2}}$. Thus, $v \in N u^{\prime} H_{i_{2}}$, so there exists some $a \in N$ and $h^{\prime} \in H_{i_{2}}$ such that $v=a u^{\prime} h^{\prime}$. However, $H_{i_{2}}=W_{>i_{2}} W_{<i_{2}}$, so we can write $h^{\prime}=g h$, where $g \in W_{>i_{2}}$ and $h \in W_{<i_{2}}$. Note that $g h=h g$, and also that $W_{>i_{2}} \leq W_{>i_{1}} \leq H_{i_{1}}$, and that, for all $j \geq 2$, we have $W_{<i_{2}} \leq W_{<i_{j}} \leq H_{i_{j}}$, that is, $h \in H_{i_{j}}$ if $j \geq 2$ and $g \in H_{i_{j}}$ if $j \leq 2$. Let $u=v g^{-1}$. Then, for $j \geq 2$, we have $N u H_{i_{j}}=N v g^{-1} H_{i_{j}}=N a u^{\prime} g h g^{-1} H_{i_{j}}=N u^{\prime} h H_{i_{j}}=N u^{\prime} H_{i_{j}}=N u_{i_{j}} H_{i_{j}}$, and, for $j \leq 2$, we have $N u H_{i_{j}}=N v g^{-1} H_{i_{j}}=N v H_{i_{j}}=N u_{i_{j}} H_{i_{j}}$. Hence we have found an element $u \in W$ with the property required to complete the induction.

Theorem 2.3. Under “ $\leq$ ”, $\mathcal{Q}$ is a poset.

Proof. The relation is reflexive and antisymmetric by definition. We may show it is transitive using the previous lemma with $m=3$, since showing transitivity for the cases involving $Q_{-1}$ or $Q_{n}$ is trivial.

Note that if we choose $N$ to be the trivial group, then the elements of the $\mathcal{Q}_{i}$ become the left cosets of the $H_{i}$, and we obtain in fact the regular polytope $\mathcal{M}(W)$ whose automorphism group $A(\mathcal{Q})$ is isomorphic to $W$. For nontrivial $N$, the map $\psi: \mathcal{M}(W) \rightarrow$ $\mathcal{Q}$ taking $u H_{i}$ to $N u H_{i}$ is a covering, so the poset $\mathcal{Q}$ may be regarded as the quotient of $\mathcal{M}(W)$ by $N$. We denote it $\mathcal{Q}=\mathcal{M}(W) / N$. If $N=\{1\}$, then $\mathcal{Q}=\mathcal{M}(W)$.

We do not explore here the conditions on $N$ under which this construction yields a well-defined polytope, since these are given by Proposition 13 of [9].

\section{The Flag Action}

We turn from quotients for the moment, and look to the general concept of abstract incidence polytopes. We let $\mathcal{P}$ be a rank $n$ incidence polytope, and let $W$, as before, be a string C-group, of type $\left[q_{1}, \ldots, q_{n-1}\right]$. The $q_{i} \geq 2$ may be infinite. At this stage we do not assume any particular relationship between $W$ and $\mathcal{P}$.

For any $\Phi \in \mathcal{F}(\mathcal{P})$ and $s_{i} \in S$, let $\Phi^{s_{i}}$ be the unique flag of $\mathcal{P}$ differing from $\Phi$ only by an element of dimension $i$, so $(\Phi)_{j}=\left(\Phi^{s_{i}}\right)_{j}$ if and only if $i \neq j$. Note that $\left(\Phi^{s_{i}}\right)_{i}$ depends only on $(\Phi)_{i-1},(\Phi)_{i}$ and $(\Phi)_{i+1}$. Given any word $w=s_{i_{1}} \cdots s_{i_{k}}$ on the 
$s_{i}$, define $\Phi^{w}$ inductively via $\Phi^{s_{i_{1}} \cdots s_{i_{k}}}=\left(\Phi^{s_{i_{1}}}\right)^{s_{i_{2}} \cdots s_{i_{k}}}$. Then, for any two words $x$ and $y$ on the $s_{i}$, we will have $\Phi^{x y}=\left(\Phi^{x}\right)^{y}$, where $x y$ is the concatenation of $x$ and $y$.

Of course, this does not define an action of $W$ on the set of flags, unless in fact, for any (defining) relation $r \equiv r^{\prime}$ of $W$, and any flag $\Phi$ of $\mathcal{P}$, we have $\Phi^{r}=\Phi^{r^{\prime}}$; for only then can we be sure that any two distinct words $w$ and $w^{\prime}$ which are equal as elements of $W$ will satisfy $\Phi^{w}=\Phi^{w^{\prime}}$. If the action is well defined, we call it the flag action of $W$ on $\mathcal{F}(\mathcal{P})$. It is sometimes also referred to as as the flag action of $W$ on $\mathcal{P}$, although strictly speaking this is an abuse of terminology. The next few results aim to determine exactly when $W$ has a flag action on $\mathcal{P}$, at least in the case when the C-group $W$ is a Coxeter group.

The defining relations of $W$ fall into four categories. Common to all C-groups there are those of the form $s_{i}^{2}=1$, those of the form $\left(s_{i} s_{j}\right)^{2}=1$, where $i \neq j, j \pm 1$, and those of the form $\left(s_{i} s_{i+1}\right)^{q_{i}}=1$, when $q_{i}<\infty$. Finally, there will be relations specific to the particular C-group in question. It is beyond the scope of this paper to examine relations of this fourth category. The next two lemmas, dealing with the first two of these forms, follow immediately from the definition of the flag action and the properties of a polytope.

Lemma 3.1. For any flag $\Phi$ and any $s_{i} \in S$, we have $\Phi^{s_{i}^{2}}=\Phi$.

Lemma 3.2. $\Phi^{s_{i} s_{j}}=\Phi^{s_{j} s_{i}}$ whenever $j \neq i, i \pm 1$.

Relations of these two types will therefore pose no problems in the definition of the flag action. The third type sometimes will, and the next lemma tells us when.

Lemma 3.3. Let $q_{i} \geq 2$ be finite, and let $\Phi$ be a flag such that $(\Phi)_{i+2} /(\Phi)_{i-1}$ is an l-gon. Then $\Phi^{\left(s_{i} s_{i+1}\right)^{q_{i}}}=\Phi$ if and only if l divides $q_{i}$.

Proof. This lemma may also be shown via a straightforward application of the definition of the flag action, and the knowledge of the structure of an $l$-cycle.

In the case where $W$ is a Coxeter group, these three lemmas immediately tell us when the flag action is well defined.

Theorem 3.4. Let $W$ be a Coxeter group, of type $\left[q_{1}, \ldots, q_{n-1}\right]$. There is a well-defined action of $W$ on $\mathcal{F}(\mathcal{P})$ satisfying $\left(\Phi^{s_{i}}\right)_{j}=(\Phi)_{j}$ if and only if, $i \neq j$, if and only if, for each $i$, either $q_{i}$ is infinite or, for all flags $\Phi$ of $\mathcal{P}, q_{i}$ is a multiple of $l$, where $(\Phi)_{i+2} /(\Phi)_{i-1}$ is an l-gon.

Note that every polytope is acted on by at least one C-group, namely, the Coxeter group of type $[\infty, \ldots, \infty]$. It would usually be more practical to work with less complicated C-groups, such as those for which the $q_{i}$ are as small as possible. A good choice would be to make $q_{i}$ the least common multiple (possibly infinite) of all $l \in \mathbf{Z}^{+} \cup\{\infty\}$ such that a flag $\Phi$ of $\mathcal{P}$ exists for which $(\Phi)_{i+2} /(\Phi)_{i-1}$ is an $l$-gon. 
From now on, let $\mathcal{P}$ be a polytope, and let $W$ be a $C$-group such that the flag action of $W$ on $\mathcal{F}(\mathcal{P})$ is well defined. This action should not be confused with the (natural) action of the automorphism group $A(\mathcal{P})$ on $\mathcal{F}(\mathcal{P})$, particularly in the case when $\mathcal{P}$ is regular. The following result is important.

Theorem 3.5. The flag action is a transitive action of $W$ on $\mathcal{F}(\mathcal{P})$.

Proof. Recall that any complex is flag-connected, so that there exists a sequence $\Phi=$ $\Phi_{1}, \Phi_{2}, \ldots, \Phi_{m}=\Phi^{\prime}$ of flags of $\mathcal{P}$ such that, for any $j$, the flags $\Phi_{j}$ and $\Phi_{j+1}$ differ by exactly one element. For each $j$, let that element have dimension $i_{j}$, so $\Phi_{j+1}=\Phi_{j}^{s_{i_{j}}}$. It follows that $\Phi_{n}=\Phi_{1}^{s_{1} s_{i_{2}} \cdots s_{i_{m-1}}}$, yielding the required result.

The next theorem clarifies how the flag action relates to the sections of a polytope. In order to avoid ambiguities in the proof, we need to introduce some more detailed notation. It will also be useful to note that, for any flag $\Phi$ of $\mathcal{P},\left(\Phi^{s_{j}}\right)_{i}$ depends only on $(\Phi)_{i}$ and (if $\left.j=i\right)(\Phi)_{i \pm 1}$.

Given an $n$-polytope $\mathcal{P}$ and a string C-group $W=\left\langle s_{0}, \ldots, s_{d-1}\right\rangle$, define $\varphi_{\mathcal{P}, W}$ to be the flag action of $W$ on $\mathcal{F}(\mathcal{P})$, so $\varphi_{\mathcal{P}, W}(\Phi, w)=\Phi^{w}$ (for $\Phi \in \mathcal{F}(\mathcal{P})$ and $w \in W$ ).

Now let $\mathcal{S}=G / F$ be a section of $\mathcal{P}$ satisfying $\operatorname{rank} F=i$ and $\operatorname{rank} G=j+1$ (so rank $\mathcal{S}=j-i$ ) and let $H$ be the subgroup $\left\langle s_{i+1}, \ldots, s_{j}\right\rangle$ of $W$. Write $s_{k}=r_{k-i-1}$, so $H=\left\langle r_{0}, \ldots, r_{j-i-1}\right\rangle$. We attempt to define actions $\alpha$ and $\beta$ of $H$ on $\mathcal{F}(\mathcal{S}$ ), via (for $\Phi \in \mathcal{F}(\mathcal{S})$ and $h \in H)$

$$
\alpha(\Phi, h)=\varphi_{\mathcal{S}, H}(\Phi, h)
$$

and

$$
\beta(\Phi, h)=\mathcal{S} \cap \varphi_{\mathcal{P}, W}\left(\Phi^{\prime}, h\right),
$$

where $\Phi^{\prime}$ is any flag of $\mathcal{P}$ which contains $\Phi$. That is, if they are well defined, $\alpha$ is the flag action of $H$ on $\mathcal{S}$, and $\beta$ is the "restriction" to $H$ and $\mathcal{F}(\mathcal{S})$ of the flag action of $W$ on $\mathcal{P}$.

Theorem 3.6. Suppose that the action of $W$ on $\mathcal{P}$ is well defined. Then the maps $\alpha$ and $\beta$ are equal and are well-defined actions of the group $H$ on the set of flags of $\mathcal{S}$.

Proof. First, let $\Phi \in \mathcal{F}(\mathcal{S})$ and $0 \leq k \leq j-i-1$, and let $\Psi=\alpha\left(\Phi, r_{k}\right)$ and $\Psi^{\prime}=\beta\left(\Phi, r_{k}\right)$. If $m \neq k$, then $(\Psi)_{m}=\left(\Psi^{\prime}\right)_{m}=(\Phi)_{m}$ by definition of the flag action (on $\mathcal{S}$ and $\mathcal{P}$, respectively). Now consider the set $\left\{F \in \mathcal{P}:(\Phi)_{k-1}<F<(\Phi)_{k+1}\right\}$. This set, which equals $\left\{F \in \mathcal{S}:(\Phi)_{k-1}<F<(\Phi)_{k+1}\right\}$, has exactly two elements. By definition of $\alpha$, these elements are $(\Phi)_{k}$ and $(\Psi)_{k}$, but by definition of $\beta$, they are $(\Phi)_{k}$ and $\left(\Psi^{\prime}\right)_{k}$, whence $(\Psi)_{k}=\left(\Phi^{\prime}\right)_{k}$, so $\Psi=\Psi^{\prime}$. It follows that, for any word $h$ on $\left\{r_{0}, \ldots, r_{j-i-1}\right\}$ and any $\Phi \in \mathcal{F}(\mathcal{S})$, we have $\varphi_{\mathcal{S}, H}(\Phi, h)=\alpha(\Phi, h)=\beta(\Phi, h)=\mathcal{S} \cap \varphi_{\mathcal{P}, W}\left(\Phi^{\prime}, h\right)$ where $\Phi^{\prime}$ is any flag of $\mathcal{P}$ containing $\Phi$. Finally, the flag action of $H$ on $\mathcal{F}(\mathcal{S})$ will be well defined, because every relation $h \equiv h^{\prime}$ of $H$ will also be a relation of $W$, whence $\varphi_{\mathcal{S}, H}(\Phi, h)=\mathcal{S} \cap \varphi_{\mathcal{P}, W}\left(\Phi^{\prime}, h\right)=\mathcal{S} \cap \varphi_{\mathcal{P}, W}\left(\Phi^{\prime}, h^{\prime}\right)=\varphi_{\mathcal{S}, H}\left(\Phi, h^{\prime}\right)$. 
This theorem is useful because it means that any results proven about the flag action of a $\mathrm{C}$-group $W$ on the set of flags of a polytope $\mathcal{P}$, may still be applied when considering the induced action of an appropriate parabolic subgroup of $W$ on the set of flags of the corresponding section of $\mathcal{P}$. Another lemma which proves useful is the following.

Lemma 3.7. Let $\Phi$ and $\Phi^{\prime}$ be flags of $\mathcal{P}$, and let $I \subseteq\{0,1, \ldots, n-1\}$ and $H_{I}=$ $\left\langle s_{i}: i \notin I\right\rangle$. Then $(\Phi)_{j}=\left(\Phi^{\prime}\right)_{j}$ for all $j \in I$ if and only if there exists some $h \in H_{I}$ such that $\Phi^{\prime}=\Phi^{h}$.

Proof. It is easy to show by induction on the length of $h$, that if $h \in H_{I}$, then $(\Phi)_{j}=$ $\left(\Phi^{h}\right)_{j}$ for all $j \in I$. To prove the converse, we use induction on the size of the set $I$. By Theorem 3.5, the result holds if $I$ is the empty set. Next, let $k \geq 1$, assume that the result holds whenever $I$ has less than $k$ elements, and let $|I|=k$. Choose $i \in I$, and let $(\Phi)_{i}=\left(\Phi^{\prime}\right)_{i}=F$. Consider the flags $\left\{(\Phi)_{-1},(\Phi)_{0}, \ldots,(\Phi)_{i}\right\}$ and $\left\{\left(\Phi^{\prime}\right)_{-1},\left(\Phi^{\prime}\right)_{0}, \ldots,\left(\Phi^{\prime}\right)_{i}\right\}$ of $F / P_{-1}$. Now, by Theorem 3.6, the group $\left\langle s_{0}, \ldots, s_{i-1}\right\rangle$ acts on the set of flags of $F / P_{-1}$ via the flag action, and, by the inductive hypothesis, there in fact exists some element $u$ of $H=\left\langle s_{j}: j<i\right.$ and $\left.j \notin I\right\rangle=W_{<i} \cap H_{I}$ with $\left\{(\Phi)_{-1}, \ldots,(\Phi)_{i}\right\}^{u}=\left\{\left(\Phi^{\prime}\right)_{-1}, \ldots,\left(\Phi^{\prime}\right)_{i}\right\}$. Note that since $H \leq W$, the element $u$ also acts on $\mathcal{F}(\mathcal{P})$, and will in fact satisfy

$$
\left(\Phi^{u}\right)_{j}= \begin{cases}\left(\Phi^{\prime}\right)_{j}, & j \leq i, \\ (\Phi)_{j}, & j \geq i .\end{cases}
$$

Similarly, we can find an element $v$ of $H^{\prime}=\left\langle s_{j}: j>i\right.$ and $\left.j \notin I\right\rangle=W_{>i} \cap H_{I}$ such that

$$
\left(\left(\Phi^{u}\right)^{v}\right)_{j}= \begin{cases}\left(\Phi^{u}\right)_{j}, & j \leq i \\ \left(\Phi^{\prime}\right)_{j}, & j \geq i\end{cases}
$$

That is, $\left(\Phi^{u v}\right)_{j}=\left(\Phi^{\prime}\right)_{j}$ for all $j \in\{0,1, \ldots, n-1\}$, and so $\Phi^{u v}=\Phi^{\prime}$. However, $w=u v$ will be an element of $\left(W_{<i} \cap H_{I}\right) \cdot\left(W_{>i} \cap H_{I}\right) \subseteq H_{I}$ as required. This completes the induction, and hence the proof.

\section{Isomorphisms and Automorphisms}

Let $\mathcal{P}$ and $\mathcal{R}$ be isomorphic polytopes. Any isomorphism $\psi$ from $\mathcal{P}$ to $\mathcal{R}$ defines, in a natural way, a bijection from $\mathcal{F}(\mathcal{P})$ to $\mathcal{F}(\mathcal{R})$, with $\Phi=\left\{(\Phi)_{i}:-1 \leq i \leq n\right\}$ being mapped to $\Phi^{\psi}=\left\{(\Phi)_{i} \psi:-1 \leq i \leq n\right\}$. If we were examining automorphisms of $\mathcal{P}$, we could say that there is an action of the automorphism group $A(\mathcal{P})$ on $\mathcal{F}(\mathcal{P})$. Also, if two isomorphisms $\psi$ and $\psi^{\prime}$ induce the same bijection, they must in fact be the same isomorphism from $\mathcal{P}$ to $\mathcal{R}$. This fact allows us to blur the distinction between $\psi$ and its induced bijection from $\mathcal{F}(\mathcal{P})$ to $\mathcal{F}(\mathcal{R})$, and also the distinction between the permutation group $A(\mathcal{P})$ of $\mathcal{P}$, and its natural isomorphic copy in the symmetric group $\operatorname{Sym} \mathcal{F}(\mathcal{P})$.

Regarded as a bijection from $\mathcal{F}(\mathcal{P})$ to $\mathcal{F}(\mathcal{R})$, an isomorphism $\psi$ from $\mathcal{P}$ to $\mathcal{R}$ will have the property of consistent projections, that is, for flags $\Phi$ and $\Phi^{\prime}$ of $\mathcal{P}$ satisfying $(\Phi)_{i}=\left(\Phi^{\prime}\right)_{i}$, it must be that $\left(\Phi^{\psi}\right)_{i}=\left(\Phi^{\prime \psi}\right)_{i}$. The following lemma is therefore an important result about isomorphisms and automorphisms. 
Lemma 4.1. Let $\mathcal{P}$ and $\mathcal{R}$ be polytopes, and let $W$ be a string $C$-group acting on them via the flag action. Further, let $\psi$ be a bijection from $\mathcal{F}(\mathcal{P})$ to $\mathcal{F}(\mathcal{R})$. Then $\psi$ has consistent projections if and only if, for all $w \in W$ and for all $\Phi \in \mathcal{F}(\mathcal{P})$, we have $\left(\Phi^{w}\right)^{\psi}=\left(\Phi^{\psi}\right)^{w}$.

Proof. Assume first that, for all $w \in W$ and for all $\Phi \in \mathcal{F}(\mathcal{P})$, we have $\left(\Phi^{w}\right)^{\psi}=$ $\left(\Phi^{\psi}\right)^{w}$. Let $\Phi, \Phi^{\prime} \in \mathcal{F}(\mathcal{P})$ with $(\Phi)_{i}=\left(\Phi^{\prime}\right)_{i}$. By Lemma 3.7, there exists $h \in H_{i}$ such that $\Phi=\Phi^{\prime h}$. Then $\left(\Phi^{\psi}\right)_{i}=\left(\left(\Phi^{\prime h}\right)^{\psi}\right)_{i}=\left(\left(\Phi^{\prime \psi}\right)^{h}\right)_{i}=\left(\Phi^{\prime \psi}\right)_{i}$, so $\psi$ has consistent projections. To show the converse, let $\psi$ be a bijection with consistent projections, let $\Phi$ be a flag of $\mathcal{P}$, and let $s_{i}$ be a generator of $W$. It is sufficient to show that $\left(\Phi^{\psi}\right)^{s_{i}}=\left(\Phi^{s_{i}}\right)^{\psi}$. If $j \neq i$, then $\left(\Phi^{s_{i}}\right)_{j}=(\Phi)_{j}$, hence $\left(\left(\Phi^{s_{i}}\right)^{\psi}\right)_{j}=\left(\Phi^{\psi}\right)_{j}=\left(\left(\Phi^{\psi}\right)^{s_{i}}\right)_{j}$. Note also that neither $\Phi^{\psi s_{i}}$ nor $\Phi^{s_{i} \psi}$ is equal to $\Phi^{\psi}$. However, the set $\left\{\Phi^{\prime} \in \mathcal{F}(\mathcal{R}):\left(\Phi^{\psi}\right)_{j}=\left(\Phi^{\prime}\right)_{j}\right.$ for all $j \neq i$ \} contains only one element not equal to $\Phi^{\psi}$. This element must be both $\Phi^{s_{i} \psi}$ and $\Phi^{\psi s_{i}}$, making these equal.

Define $\Gamma(\mathcal{P})=W^{\mathcal{F}(\mathcal{P})}$ to be the subgroup of $\operatorname{Sym}(\mathcal{F}(\mathcal{P}))$ induced by the flag action. (It may be shown that, for a regular polytope, this $\Gamma(\mathcal{P})$ is isomorphic to the automorphism group $A(\mathcal{P})$.) A corollary to the lemma we have just shown is that the centralizer of $\Gamma(\mathcal{P})$ is the set of all permutations of $\mathcal{F}(\mathcal{P})$ with consistent projections, which is a superset of $A(\mathcal{P})$. The next theorem makes the link between $\Gamma(\mathcal{P})$ and $A(\mathcal{P})$ even more explicit. Note first that since the centralizer is a group, if $\alpha \in \operatorname{Sym}(\mathcal{F}(\mathcal{P}))$ has consistent projections, so will $\alpha^{-1}$.

Theorem 4.2. If $\alpha^{\prime} \in \operatorname{Sym}(\mathcal{F}(\mathcal{P}))$ has consistent projections, it induces an automorphism of $\mathcal{P}$.

Proof. Given such an $\alpha^{\prime}$, we define a permutation $\alpha$ of $\mathcal{P}$ as follows. For $F \in \mathcal{P}$ with rank $F=i$, choose $\Phi \in \mathcal{F}(\mathcal{P})$ with $(\Phi)_{i}=F$. Then let $F \alpha=\left(\Phi^{\alpha^{\prime}}\right)_{i}$. This will not depend on the choice of $\Phi$. Now $\alpha^{\prime-1}$ induces another permutation of $\mathcal{P}$, which will serve as an inverse for $\alpha$, so $\alpha$ is a bijection from $\mathcal{P}$ to itself. Finally, for $F, G \in \mathcal{P}$, we can show that $F \leq G$ if and only if $F \alpha \leq G \alpha$ by applying $\alpha^{\prime}$ to any flag of $\mathcal{P}$ containing both $F$ and $G$, or $\alpha^{\prime-1}$ to any flag containing $F \alpha$ and $G \alpha$.

Thus $A(\mathcal{P})$ is the centralizer of $\Gamma(\mathcal{P})$, when regarded as a subgroup of $\operatorname{Sym}(\mathcal{F}(\mathcal{P}))$.

Note that Theorem 4.2 is not trivial. Although it holds for incidence polytopes, it is not trivial to prove, and cannot be extended to arbitrary incidence complexes. See, for instance, the example following Lemma 3.4.2 of [4].

\section{The Main Results}

Let $\mathcal{P}$ be an $n$-incidence polytope, and let $W$ be a C-group acting on $\mathcal{F}(\mathcal{P})$ via the flag action. Let $\mathcal{M}=\mathcal{M}(W)$ be the regular polytope with group $W$. Select and fix a flag $\Phi$ of $\mathcal{P}$. We may consider $\Phi$ to be a "base flag" for what follows. Let $N$ be equal to $\left\{a \in W: \Phi^{a}=\Phi\right\}$, the stabilizer $W_{\Phi}$ of $\Phi$ in the group $W$, and let $\mathcal{Q}=\mathcal{M} / N$ be the 
quotient of $\mathcal{M}$ by $N$, as defined in Section 2. We shall define a map $\Omega$ from $\mathcal{P}$ to $\mathcal{M} / N$, and (in Theorem 5.2) show it to be an isomorphism.

First, define $\Omega^{*}$ to be a map taking elements of $\mathcal{P} \backslash\left\{P_{-1}, P_{n}\right\}$ to certain subsets of $W$, as follows. For $F \in \mathcal{P} \backslash\left\{P_{-1}, P_{n}\right\}$, let $w \in F \Omega^{*}$ if and only if $F \in \Phi^{w}$; that is (if rank $F=i$, we have $w \in F \Omega^{*}$ if and only if $F=\left(\Phi^{w}\right)_{i}$. Our aim will be to use $\Omega^{*}$ to construct an isomorphism $\Omega$ from $\mathcal{P}$ to $\mathcal{M} / N$. The following lemma tells us what the subsets $f \Omega^{*}$ will be.

Lemma 5.1. If $F \in \mathcal{P}_{i}(0 \leq i \leq n-1)$ and if $w \in F \Omega^{*}$, then $F \Omega^{*}=N w H_{i}$.

Proof. Let $F$ and $w$ be as in the statement of the lemma, and let $u \in N w H_{i}$. Then $u=a w h$, for some $a \in N$ and $h \in H_{i}$. Now $\left(\Phi^{u}\right)_{i}=\left(\Phi^{a w h}\right)_{i}=\left(\Phi^{w h}\right)_{i}$, since $\Phi^{a}=\Phi$. However, $\left(\Phi^{w h}\right)_{i}=\left(\Phi^{w}\right)_{i}$, since $h \in H_{i}$ (see Lemma 3.7), and $\left(\Phi^{w}\right)_{i}=F$, since $w \in F \Omega^{*}$ and $\operatorname{rank} F=i$. Thus $\left(\Phi^{u}\right)_{i}=F$ whence $u \in F \Omega^{*}$. Conversely, let $u \in F \Omega^{*}$, so $\left(\Phi^{u}\right)_{i}=F=\left(\Phi^{w}\right)_{i}$. Lemma 3.7 informs us, since $\left(\Phi^{u}\right)_{i}=\left(\Phi^{w}\right)_{i}$, that there exists $h \in H_{i}$ with $\Phi^{w}=\Phi^{u h}$, that is, $\Phi=\Phi^{u h w^{-1}}$. It follows that $u h w^{-1} \in N$, so $u \in N w h^{-1}$, whence in fact $u \in N w H_{i}$. This yields the required equality $F \Omega^{*}=$ $N w H_{i}$.

Now, we define the map $\Omega$ from $\mathcal{P}$ to $\mathcal{Q}=\mathcal{M} / N$ as follows. Let $P_{-1} \Omega=Q_{-1}$ and $P_{n} \Omega=Q_{n}$, and let $F \Omega=F \Omega^{*}$ for all other $F \in \mathcal{P}$.

Theorem 5.2. $\Omega$ is an isomorphism from $\mathcal{P}$ to $\mathcal{M} / N$.

Proof. Lemma 5.1 tells us that it is a well-defined map. We have to show now that it is a structure-preserving bijection. To show that it is one to one, let $F \Omega=G \Omega=N u H_{i}$ for some $F, G \in \mathcal{P}$ and $N u H_{i} \in \mathcal{M} / N$. If $\operatorname{rank} F=j \neq i$, then since $u \in N u H_{i}=F \Omega$, it must be that $F \Omega=N u H_{j}$ (Lemma 5.1). Then we would have $N u H_{i}=N u H_{j}=$ $W$ (Lemma 2.1). In particular, $\left\{1, s_{j}\right\} \subseteq N u H_{j}$, so $(\Phi)_{j}=\left(\Phi^{s_{j}}\right)_{j}=F$. This is a contradiction, so in fact rank $F=i$. Similarly, rank $G=i$. Then, if $F \Omega=G \Omega=N u H_{i}$, we have $\left(\Phi^{u}\right)_{i}$ equal to both $F$ and $G$, yielding $F=G$; thus $\Omega$ is one to one. To show that it is onto, let $N u H_{i} \in \mathcal{M} / N$, and let $F=\left(\Phi^{u}\right)_{i}$. Then, by definition, $u \in F \Omega$, and so, by the previous lemma, $F \Omega=N u H_{i}$, as required. Finally, $\Omega$ preserves the partial order: first, let $F, G \in \mathcal{P}$ with $F \leq G$, let $\Phi^{\prime}$ be a flag containing both $F$ and $G$, and let $i \leq j$ be such that $i=\operatorname{rank} F$ and $j=\operatorname{rank} G$. By Theorem 3.5, there exists $u \in W$ such that $\Phi^{u}=\Phi^{\prime}$. Then $\left(\Phi^{u}\right)_{i}=F$ and $\left(\Phi^{u}\right)_{j}=G$, so $F \Omega=N u H_{i}$ and $G \Omega=N u H_{j}$, whence $F \Omega \cap G \Omega$ is nonempty. It follows that $F \Omega \leq G \Omega$ as required. Conversely, let $F, G \in \mathcal{P}$ be such that $F \Omega \leq G \Omega$. Then $F \Omega \cap G \Omega$ is nonempty, and $i \leq j$ (where rank $F=i$ and rank $G=j$ ). Letting $u$ be an element of $F \Omega \cap G \Omega$, it follows that $F \Omega=N u H_{i}$ and $G \Omega=N u H_{j}$. Thus $F=\left(\Phi^{u}\right)_{i}$ and $G=\left(\Phi^{u}\right)_{j}$, so $F$ and $G$ are both elements of the flag $\Phi^{u}$. It follows that either $F \leq G$ or $G \leq F$. We conclude the former, since $i \leq j$. This completes the proof.

It follows that any polytope $\mathcal{P}$ is isomorphic to some kind of quotient. Note that in the above proof, the group $N$ defining the quotient was found to have the property that $N u H_{i} \neq N v H_{j}$ whenever $i \neq j$. This further justifies the comments made in Section 2 about treating the $\mathcal{Q}_{i}$ as disjoint sets. 
Theorem 5.3. Let $\mathcal{P}$ and $\mathcal{R}$ be polytopes acted on by the $C$-group $W$ via the flag action, and let $N=W_{\Phi}$ and $N^{\prime}=W_{\Phi^{\prime}}$ for some flags $\Phi \in \mathcal{F}(\mathcal{P})$ and $\Phi^{\prime} \in \mathcal{F}(\mathcal{R})$. Then $\mathcal{P}$ and $\mathcal{R}$ are isomorphic if and only if $N$ and $N^{\prime}$ are conjugate.

Proof. Assume first that $\mathcal{P}$ and $\mathcal{R}$ are isomorphic, and let $\psi$ be an isomorphism from $\mathcal{P}$ to $\mathcal{R}$. Now $\Phi^{\psi} \in \mathcal{F}(\mathcal{R})$, so Theorem 3.5 tells us that there exists $u \in W$ such that $\left(\Phi^{\prime}\right)^{u}=\Phi^{\psi}$, so $\Phi^{\prime}=\Phi^{\psi u^{-1}}$. Now $a \in N$ if and only if $\Phi^{a}=\Phi$. By Lemma 4.1, $\Phi^{a \psi}=\Phi^{\psi a}$, so $a \in N$ if and only if $\Phi^{\prime u a u^{-1}}=\Phi^{\psi a u^{-1}}=\Phi^{a \psi u^{-1}}=\Phi^{\psi u^{-1}}=\Phi^{\prime}$. Thus $a \in N$ if and only if $u a u^{-1} \in N^{\prime}$, whence $N^{\prime}=u N u^{-1}$ as required. Conversely, let $N^{\prime}=u N u^{-1}$. We construct the map $\varphi$ from $\mathcal{M} / N$ to $\mathcal{M} / N^{\prime}$ via $\left(N w H_{i}\right) \varphi=N^{\prime} u w H_{i}$. It is then not hard to show that $\varphi$ is a well-defined, structure-preserving bijection, whence $\mathcal{P} \cong \mathcal{M} / N \cong \mathcal{M} / N^{\prime} \cong \mathcal{R}$ as required.

We may say, then, that any polytope is a quotient, of the form $\mathcal{M} / N$ for some $N \leq W$, and two polytopes are isomorphic if and only if they are quotients $\mathcal{M} / N$ and $\mathcal{M} / N^{\prime}$ by conjugate subgroups $N$ and $N^{\prime}$ of $W$. It follows that the theory of (even nonregular) incidence polytopes may be expressed in terms of the theory of C-groups (or just Coxeter groups) and their subgroups.

\section{Acknowledgements}

The author wishes to thank Egon Schulte for bringing certain key references to his attention, and Cheryl Praeger, Robert Howlett and Egon Schulte again for their valuable comments on some early drafts of this paper. The comments by the (anonymous) referee were also very helpful.

\section{References}

1. Bourbaki, N.: Groupes et Algèbres de Lie, Chapters IV-VI. Hermann, Strasbourg, 1968.

2. Danzer, L., Schulte, E.: Reguläre Inzidenzkomplexe I. Geom. Dedicata 13, 295-308 (1982).

3. Davis, M.W.: Regular Convex Cell Complexes. In: McCrory, C. et al.: Geometry and Topology, pp. 53-88. Lecture Notes in Pure and Applied Mathematics, vol. 105. Marcel Dekker, New York, 1987.

4. Hartley, M.I.: Combinatorially Regular Euler Polytopes. Ph.D. thesis, University of Western Australia, 1996.

5. Humphreys, J.E.: Reflection Groups and Coxeter Groups. Cambridge University Press, Cambridge, 1990.

6. Kato, M.: On Combinatorial Space Forms. Sci. Papers College Gen. Ed. Univ. Tokyo 30, 107-146 (1980).

7. McMullen, P., Schulte, E.: Regular Polytopes from Twisted Coxeter Groups and Unitary Reflection Groups. Adv. in Math. 82, 35-87 (1990).

8. McMullen, P., Schulte, E.: Hermitian Forms and Locally Toroidal Regular Polytopes. Adv. in Math. 82, 88-125 (1990).

9. McMullen, P., Schulte, E.: Quotients of Polytopes and C-Groups. Discrete Comput. Geom. 11, 453-464 (1994).

10. Schulte, E.: Reguläre Inzidenzkomplexe. Dissertation, University of Dortmund, 1980.

11. Tits, J.: A Local Approach to Buildings. In: Davis, C. et al.: The Geometric Vein: The Coxeter Festschrift, Springer-Verlag, New York, 1980.

Received January 27, 1997, and in revised form October 1, 1997. 